\title{
Tangibilidade e Intangibilidade na Determinação do Desempenho Persistente de Firmas Brasileiras
}

\section{Tangibility and Intangibility in Determining the Persistent Performance of Brazilian Firms}

Francisval de Melo Carvalho * Doutor em Administração pela Universidade Presbiteriana Mackenzie. Professor Adjunto da UFLA, Lavras/MG, Brasil.

Eduardo Kazuo Kayo Doutor em Administração pela FEA/USP. Professor da FEA/USP, São Paulo/SP, Brasil.

Diógenes Manoel Leiva Martin Doutor em Administração pela EAESP/FGV. Professor do PPGA/Universidade Presbiteriana Mackenzie, São Paulo/SP, Brasil.

* Endereço: Francisval de Melo Carvalho

DAE, Campus Universitário da UFLA, Lavras/MG, 37200-000. E-mail: francarv@dae.ufla.br

Copyright (C) 2010 RAC. Todos os direitos, inclusive de tradução, são reservados. É permitido citar parte de artigos sem autorização prévia desde que seja identificada a fonte. 


\section{RESUMO}

Preconiza a RBV que os recursos e competências são distribuídos de forma heterogênea entre as firmas, sendo a principal causa da variação observada entre seus desempenhos. Quando esses recursos são raros, valiosos e difíceis de serem imitados e a firma dispõe de estrutura organizacional adequada para explorá-los, eles têm o potencial de promover desempenho acima da média e persistente. O objetivo deste artigo é avaliar se o desempenho persistente das firmas tem alguma relação com a intangibilidade ou tangibilidade dos seus recursos. A amostra foi constituída por empresas brasileiras de capital aberto com ações listadas na Bovespa. A técnica estatística utilizada foi o painel dinâmico com estimativas pelo Método dos Momentos Generalizados. Os resultados mostram que, para as empresas brasileiras analisadas, a intangibilidade dos recursos não se revelou como vantagem competitiva sustentável, pois foi constatado que esses recursos não contribuem para a persistência do desempenho superior das firmas. Por outro lado, a tangibilidade dos recursos contribuiu de forma significativa para a persistência do desempenho superior das firmas de diversos setores da economia.

Palavras-chave: desempenho persistente; intangibilidade de recursos; tangibilidade de recursos; vantagem competitiva sustentável.

\section{ABSTRACT}

RBV states that resources and capabilities are heterogeneously distributed among firms and are the main drivers of their different performances. When these resources are rare, valuable and difficult to imitate and the firm has a suitable organizational structure for exploiting them, they have the potential to lead the firms to a persistent, above average performance. The aim of this article is to analyze whether the firm's persistent performance is related to the intangibility or tangibility of their resources. The sample comprises Brazilian public companies listed on the Bovespa Stock Exchange in São Paulo. The statistical technique employed was the dynamic panel data, with estimates obtained by the generalized method of moments. The results show that, in the case of the Brazilian companies under study, the intangibility of resources was not a sustainable competitive advantage because these resources do not persistently contribute to the higher performance of firms. On the other hand, the tangibility of resources played a significant contribution to the persistent performance of firm operating in different industries.

Key words: persistent performance; intangibility of resources; tangibility of resources; sustainable competitive advantage. 


\section{INTRODUÇÃO}

Existem evidências teóricas e empíricas de que fatores idiossincráticos, específicos de cada firma individual, têm papel preponderante na explicação da variação do desempenho das firmas. Essa é a posição da Resource Based View [RBV], ou teoria baseada nos recursos; ela preconiza que a principal causa da variação observada no desempenho das firmas está relacionada à natureza específica de seus recursos e competências acumuladas. Para essa abordagem, os recursos e competências são distribuídos de forma heterogênea entre as firmas de determinada indústria, como resultado das suas diferentes trajetórias experimentadas. Uma vez que sejam de difícil replicação (aquisição ou substituição), cria-se o potencial para o estabelecimento de vantagens competitivas que podem dar origem a desempenho superior e sustentável, até que empresas rivais obtenham um conjunto de recursos equivalentes.

Dentre os recursos que podem promover o desempenho superior das firmas se encontram os intangíveis. Na medida em que as empresas têm acesso relativamente fácil à aquisição de ativos tangíveis (terrenos, edifícios, máquinas, equipamentos, entre outros), a carteira de intangíveis passa a ser importante fator de diferenciação, contribuindo decididamente para o aumento da criação de valor das firmas. O potencial dos ativos intangíveis para criar riquezas para as empresas está diretamente relacionado a determinados atributos desses ativos. Devido às suas características e peculiaridades, na sua maioria, eles não são comercializáveis nos mercados de fatores e, em muitas situações, precisam ser desenvolvidos no interior da empresa. O processo de desenvolvimento interno precisa ser contínuo; não raro, os esforços são incertos. Esse processo pode tornar os recursos específicos da firma, o que os torna raros e de difícil imitação, com potencial significativo para a obtenção de desempenho superior e sustentável. Esse fenômeno pode fazer com que o valor de mercado de uma firma seja significativamente maior que seu valor contábil, o que aumenta, entre outros fatores, seu nível de intangibilidade. Com efeito, Lev (2001) chama atenção para o fato de que o índice de valor de mercado sobre valor contábil das 500 maiores empresas dos Estados Unidos aumentou aproximadamente seis vezes do início da década de 1980 ao ano 2001.

Se existem evidências da influência dos ativos intangíveis na obtenção de desempenho superior das firmas, por outro lado alguns estudos evidenciam o reflexo das decisões de investimento de capital, mais especificamente em recursos tangíveis, na expectativa de geração de fluxo de caixa futuro. McConnell e Muscarella (1985), por exemplo, concluíram que anúncios de aumento ou de redução de dispêndios de capital das empresas industriais estão associados, respectivamente, com aumentos ou reduções estatisticamente significativos no valor de mercado da ação. Os autores ressaltam que a valorização no mercado é válida para empresas que possuem oportunidades de investimento com valor presente líquido positivo. Resultado semelhante foi encontrado por Lucchesi e Famá (2007) para empresas brasileiras, mostrando que anúncios de investimento levam a retornos positivos nos preços das ações. Outros autores investigaram a correlação entre a variação do ativo permanente contábil e o preço de mercado das ações. Lamb (1993) encontrou resultados que revelam a existência de indícios de correlação positiva entre a variação do ativo permanente e o preço das ações. Da mesma forma, Antunes e Procianoy (2003), estudando as empresas no mercado brasileiro, encontraram evidências de uma relação entre as variações do ativo permanente e imobilizado e o preço da ação no mercado de capitais.

Se, por um lado, o valor de mercado das ações das firmas normalmente aumenta como efeito de investimentos em ativos tangíveis e intangíveis, por outro lado os efeitos sobre o desempenho contábil (exemplo: retorno sobre ativos) são menos conclusivos. Alguns estudos (exemplo: Villalonga, 2004) mostram relações positivas entre intangibilidade e desempenho contábil, ao passo que outros (exemplo: Morbey \& Reithner, 1990) apresentam relações negativas. Essa diversidade de resultados é devida, particularmente, à utilização de diferentes medidas de desempenho. Como sugerem Carneiro e Dib (2006), problemas de mensuração do constructo (nesse caso o desempenho) podem levar a resultados inconsistentes. Porém, a despeito desse problema de operacionalização, a escolha da medida de desempenho mais apropriada depende do problema de pesquisa. No presente artigo, opta-se 
pela utilização de uma medida contábil de desempenho, porém relativizada pelo setor de atuação de cada firma. O interesse, pois, é analisar o efeito da natureza dos recursos (intangibilidade/tangibilidade) sobre o desempenho de cada firma em relação aos seus concorrentes. Nesse contexto, o presente artigo propõe responder ao seguinte problema de pesquisa: Qual a influência da intangibilidade e da tangibilidade dos recursos das firmas brasileiras de capital aberto sobre seus desempenhos persistentes?

O restante do artigo está estruturado da seguinte forma. O segundo tópico apresenta a fundamentação teórica do artigo, destacando a influência dos recursos tangíveis e intangíveis sobre o desempenho superior e persistente das firmas. O terceiro tópico descreve os procedimentos metodológicos adotados na condução da pesquisa empírica, cujos resultados são apresentados no quarto tópico. O quinto tópico, por fim, tece as considerações finais do artigo.

\section{FUNDAMENTAÇÃO TEÓRICA}

\section{Desempenho Superior e Persistente}

As firmas e os setores não são homogêneos, mas apresentam diversas fontes de variação que podem interferir diretamente nos seus desempenhos. Diante desta constatação, Brito e Vasconcelos (2004a) ressaltam que o equilíbrio econômico, preconizado pelo modelo de competição perfeita, está longe de ser atingido.

Várias correntes teóricas têm procurado estudar a heterogeneidade do desempenho das firmas. March e Sutton (1997) ressaltam que a ênfase no estudo do desempenho como variável dependente é mais explícita no campo da estratégia; mas a idéia de que o desempenho deve ser previsto, entendido e determinado está presente em todas as áreas de estudos organizacionais. Glick, Washburn e Miller (2005) fizeram um levantamento de como se tratava do tema nos principais periódicos, no campo da administração estratégica, e concluíram que o desempenho da firma é tema central na administração estratégica. No entanto, a conceituação e a medida do desempenho permanecem problemáticas. Carneiro e Dib (2006) também concordam com essa colocação e ressaltam que resultados conflitantes sobre os determinantes que promovem desempenho das firmas têm sido encontrados nos estudos que investigam o tema. Para os autores, uma das possíveis causas desses resultados conflitantes é a pobreza na conceituação, operacionalização e mensuração do constructo para medir o desempenho das firmas, o que traz inconsistência nos resultados.

A utilização de indicadores financeiros para mensurar desempenho é defendida por pesquisadores que relacionam desempenho com criação de valor. Dessa forma, a verificação do efetivo agregado de valor aos acionistas deve estar relacionada a alguma métrica que permita estabelecer uma avaliação da firma. Nesse sentido, Acquaah (2003) destaca que, nos estudos que envolvem a RBV, existe uma prevalência da utilização de indicadores de desempenho padronizados, tais como retorno sobre investimento, retorno sobre ativos e fluxo de caixa líquido. Mesmo reconhecendo a complexidade da mensuração do desempenho das empresas, uma vez que este pode ser mensurado de diferentes formas, El-Shishini (2001) e Omaki (2005) ressaltam que medidas financeiras de desempenho ainda são mais usadas que outros tipos de medidas não-financeiras, particularmente porque aquelas seriam estimativas mais razoáveis do desempenho organizacional.

\section{Fatores que Exercem Influência no Desempenho das Firmas}

Identificar as fontes das diferenças entre as empresas tem sido objeto de estudo de diversos pesquisadores das áreas de economia, finanças e estratégia. Esses estudiosos têm focado em unidades de análise diferentes. Enquanto os economistas têm considerado, nos seus estudos, a indústria como 
unidade de análise dominante, os da área de estratégia de negócios têm procurado as fontes de variação e diferenças de desempenho nos aspectos internos da firma (Rumelt, 1991).

Dentre as propostas metodológicas utilizadas para estudar os fatores que afetam o desempenho da firma encontra-se a de Schmalensee (1985). Este autor estudou a influência do setor econômico e da participação do mercado na variância da lucratividade das empresas, tendo apenas os dados de um ano sido considerados. Apesar de não ser o pioneiro, o trabalho de Rumelt (1991) é o mais conhecido do gênero. O modelo de Rumelt caracteriza-se por decompor os componentes da variância da taxa de retorno de empresas, o que permite verificar qual a contribuição dos diversos fatores estudados (da indústria, da empresa individual, do ano e efeitos da corporação) no desempenho da firma. O modelo e a base de dados utilizados por Rumelt foram semelhantes aos que Schmalensee (1985) utilizou. Mas, além de adequações nos fatores analisados, o que diferenciou de forma marcante o trabalho dos dois foi a ampliação do prazo estudado de um para quatro anos, o que permitiu incluir os efeitos relativos a todo um ciclo de negócios. Os principais resultados obtidos por Rumelt (1991) são de que 46\% da variância total é explicada pelos efeitos da firma e que apenas $8 \%$ da variância dos retornos nas firmas é devido aos efeitos estáveis da indústria.

\section{Relação entre Desempenho Superior e Vantagem Competitiva}

Embora o uso do termo vantagem competitiva esteja disseminado nas diversas áreas da economia, estratégia e administração em geral, não existe consenso sobre sua definição. Percebe-se, no entanto, que a criação de valor é o tema comum relacionado à vantagem competitiva. Brito e Vasconcelos (2004b) ressaltam que o conceito está, sem dúvida, ligado a desempenho superior das empresas.

Segundo Rumelt (2003), existem várias definições que tratam do termo vantangem competitiva. Peteraf (1993) define vantagem competitiva como sendo retornos sustentados acima do normal. Barney (2002) sustenta que uma firma experimenta vantagem competitiva quando suas ações na indústria ou no mercado criam valor econômico e quando poucas firmas competidoras estão engajadas em ações similares. Relaciona vantagem competitiva a desempenho, argumentando que uma firma obtém desempenho acima do normal quando valor acima do esperado no emprego dos recursos é conseguido. Ghemawat e Rivkin (1999) colocam que uma firma que obtém, dentro da indústria ou de seu grupo estratégico, retornos financeiros superiores no longo prazo, desfruta de vantagem competitiva sobre seus rivais. Besanko, Dranove, Shanley e Schaefer (2006, p. 238) afirmam: quando uma firma obtém taxas de lucro econômico mais altas do que o índice médio de lucro econômico de outras firmas concorrentes, dentro do mesmo mercado, ela tem vantagem competitiva naquele mercado.

Percebe-se que a questão central da vantagem competitiva é que ela está relacionada diretamente com o desempenho superior da firma em relação às suas rivais, em determinado mercado. Esse desempenho superior pode ser obtido por meio da utilização de recursos ou capacidades específicas não disponíveis para aquisição no mercado. Dessa forma, a utilização pela firma desses recursos e capacidades vai redundar em retornos financeiros superiores ou aumento da participação das vendas naquele determinado mercado, em relação aos seus concorrentes. Neste trabalho, é adotada a proposta de Peteraf (1993); ele considera que obter vantagem competitiva significa alcançar resultado financeiro superior ao da média do setor de atividades no qual a empresa se insere.

A obtenção da vantagem competitiva não é o suficiente para garantir um desempenho superior da firma. A sustentabilidade dela, ao longo do tempo, é condição essencial. Nesse sentido, Bharadwaj, Varadarajan e Fahy (1993) comentam que a busca de vantagem competitiva sustentável não é fim em si, mas meio para um fim, ou seja, desempenho financeiro superior a longo prazo. Alguns autores denominam desempenho financeiro superior no longo prazo como desempenho sustentável ou como desempenho persistente. No presente trabalho será adotado o termo desempenho persistente, que consiste no desempenho financeiro da firma acima da média do setor do qual ela faz parte, no longo prazo. 


\section{Recursos da Firma como Fatores que Influenciam o Desempenho}

A percepção de que a firma é composta por uma coleção de recursos inspirou a criação de uma abordagem teórica (RBV), que tem nos recursos internos da firma a resposta para o desempenho diferenciado das empresas. Nesse sentido, a empresa passa a ser pensada como sendo o resultado das potencialidades e restrições de sua carteira de recursos e não apenas de sua carteira de produtos, uma vez que cada produto é, em última análise, resultado da combinação de recursos (Saraiva, 2007).

O trabalho de Penrose (1959) é considerado um dos inspiradores da RBV, contribuindo com a formulação de importantes conceitos, tais como: i) uma firma pode ser vista como uma coleção de recursos; ii) o caminho de crescimento de uma determinada firma pode ser otimizado, requerendo a combinação de recursos internos e externos à firma, numa sequência particular; e iii) o processo de crescimento de uma firma é dependente de suas características de gestão, da sua experiência adquirida e da capacidade de aprendizado (Rugman \& Verbeke, 2002).

Na perspectiva da RBV, os recursos desenvolvidos e controlados pelas empresas são o núcleo central para explicar o desempenho superior da firma. Dessa forma, eles são entendidos como a unidade fundamental de geração de valor (Mathews, 2002). Caves (1984) considera como recursos os ativos tangíveis e intangíveis que estejam vinculados de forma semipermanente à empresa. Atributos, como marcas, reputação, conhecimento tecnológico desenvolvido internamente, informação acumulada sobre clientes, força de trabalho especializada, habilidade gerencial, contratos, equipamentos, cultura organizacional, processos eficientes e recursos financeiros, entre outros, podem ser considerados recursos de uma empresa (Amit \& Schoemaker, 1993; Wernerfelt, 1984).

\section{Ativos Intangíveis e Desempenho Superior}

Lev (2001, p. 1) ressalta que riqueza e crescimento, na economia atual, são dirigidos, principalmente, por ativos intangíveis e que ativos físicos e financeiros se transformam rapidamente em commodities que remuneram o investimento na média, enquanto retornos acima do normal ou posição competitiva dominante são obtidos pelo desenvolvimento de ativos intangíveis acompanhados de outros tipos de ativos. Com efeito, Danthine e Jin (2007) lembram que o capital intangível resulta de investimentos em novos produtos, em publicidade, em pesquisa e desenvolvimento, em softwares, além do desenvolvimento humano por meio de treinamento, escolarização e aprendizado na prática (on-the-job learning).

A construção de uma definição adequada de ativos intangíveis é bastante complexa. Com o interesse crescente no assunto em questão, vários enfoques têm sido desenvolvidos por diferentes correntes teóricas. Enquanto na perspectiva contábil a definição de ativos parece ser mais restrita, na perspectiva econômica ela é mais abrangente, o que parece ser mais adequado aos propósitos deste artigo. Nessa análise mais abrangente, Martins (1972, p. 30), apresenta a seguinte definição: “ativo é o futuro resultado econômico que se espera obter de um agente”.

Esse conceito é consistente com o método de fluxo de caixa descontado, pelo qual o valor de um ativo (ou capital) é obtido pela soma dos fluxos de caixa futuros, descontados a uma taxa apropriada ao seu nível de risco (Yong \& O’Byrne, 2003, p. 35).

No campo da estratégia, mais especificamente na abordagem da RBV, os ativos são denominados recursos. Dessa forma, os recursos à disposição da firma incluem tanto os tangíveis, que possuem características físicas, quanto os intangíveis, que não as possuem (Helfat \& Peteraf, 2003; Wernerfelt, 1984). Villalonga (2004), sumariando conceitos de diversos autores que contribuem para a RBV, considera que os recursos intangíveis são tipicamente tácitos e difíceis de codificar. São passíveis de negociação em mercados imperfeitos de fatores e exibem complementaridades. Como consequência, torna-se difícil a aquisição, o desenvolvimento ou a replicagem desses recursos que, normalmente, são desenvolvidos internamente nas firmas. Pelas suas características, são difíceis de serem entendidos e 
copiados pelos concorrentes, tendendo a tornarem-se específicos da firma. Essa dificuldade de imitação é que os torna valiosos e os transformam na base da vantagem competitiva da empresa.

Inúmeros estudos corroboram a hipótese de que o investimento em ativos (ou recursos) intangíveis promove desempenho financeiro superior e criação de valor. Alguns autores, como Chauvin e Hirschey (1993), Megna e Klock (1993) e Connolly e Hirschey (2005), encontraram relações positivas e significantes entre o investimento em recursos relacionados à inovação e o valor de mercado das firmas. Em geral, estudos que analisam esse tipo de relacionamento utilizam variáveis como despesas com P\&D (pesquisa e desenvolvimento), quantidade de patentes, entre outras variáveis representativas da capacidade de inovação das firmas. Villalonga (2004), por sua vez, demonstra uma significativa influência da intangibilidade das firmas norte-americanas sobre o seu desempenho superior (medido pelo retorno sobre o ativo de cada firma em relação à média do setor); além disso, também constata que a intangibilidade é responsável por desempenho persistente ao longo do tempo.

Entretanto, estudos recentes sugerem que a relação entre inovação e criação de valor pode ser nãomonotônica. Lee e Chen (2009), por exemplo, observaram que as despesas com P\&D podem afetar o valor da firma de duas formas. Eles desenvolveram um estudo de evento em que analisaram o efeito de anúncios de P\&D sobre o retorno anormal da ação em determinada janela de tempo. Eles observaram que os retornos anormais das ações eram negativos, quando as firmas anunciavam níveis baixos ou moderados de P\&D. Por outro lado, os retornos eram positivos, quando o dispêndio com P\&D era alto. A possível explicação para esse fenômeno, segundo Lee e Chen (2009), era a percepção que os investidores tinham de que baixos investimentos em P\&D apenas reduziriam o lucro corrente, sem necessariamente gerar aumento no fluxo de caixa futuro. Altos níveis de investimento, por outro lado, estariam associados a importantes opções de crescimento e geração de valor no futuro.

Outro ativo intangível que contribui para a criação de valor das firmas é a marca. A marca é um dos recursos responsáveis pela determinação da preferência dos consumidores e pela percepção que estes desenvolvem pelo produto ou serviço. Com efeito, marca forte pode gerar maior lealdade dos clientes, menor vulnerabilidade a ações de marketing dos concorrentes, maiores margens de lucro, entre outras importantes consequências (Keller, 1998). Além disso, as despesas com propaganda e publicidade responsáveis pela criação nos clientes de maior reconhecimento dos produtos e serviços, também servem como instrumentos para construir imagem forte de marca por meio da dramatização e da apresentação do produto e serviço de tal forma que chame a atenção do consumidor (Ali Shah \& Akbar, 2008). Nesse contexto, autores como Chauvin e Hirschey (1993) e Barth, Clement, Foster e Kasznik (1998) já demonstraram que investimentos na formação de uma marca forte por meio de investimentos em propaganda e publicidade são positivamente associados à criação de valor. Ali Shah e Akbar (2008) apresentam ampla revisão bibliográfica dos efeitos das despesas com propaganda e publicidades sobre o desempenho das firmas, seja este medido em termos contábeis ou valor de mercado.

O potencial dos ativos intangíveis para criar riquezas para as empresas está diretamente relacionado a determinados atributos peculiares desses ativos. Nesse sentido, Lev (2001, pp. 21-49) considera que os principais benefícios associados aos intangíveis decorrem das seguintes características: efeitos de externalidade de rede, não-rivalidade e escalabilidade ilimitada. A externalidade de rede acontece quando "a demanda exercida por uma pessoa pode vir a ser influenciada pelo número de outros consumidores que já tenham adquirido a mercadoria” (Pindyck \& Rubinfeld, 1994, p. 150). A nãorivalidade refere-se à característica presente na maior parte dos ativos intangíveis de possuir usos alternativos não-rivais. Dessa forma, um ativo pode ser utilizado simultaneamente e em aplicações repetitivas, sem diminuir sua utilidade. Escalabilidade ilimitada: os ativos intangíveis, diferentemente dos ativos físicos, apresentam retorno por escala crescente, beneficiando-se cada vez mais da ampliação do volume. Dessa forma, é o tamanho do mercado que limita a escalabilidade de tais ativos.

\section{Ativos Tangíveis e Criação de Valor}

Na perspectiva do campo da estratégia, os ativos são considerados recursos. Nesse sentido, Barney 
(1991), em meio a tantas nomenclaturas existentes, propôs uma classificação pela qual os recursos de capital físico, tais como tecnologia física (equipamentos), fábricas, localização geográfica, reservas de matéria prima, entre outros, foram classificados como ativos tangíveis. Wilk (2006, p. 27) define que "Recursos tangíveis são todos aqueles que podem ser efetivamente observados e medidos, ou seja, possuem uma natureza física e/ou mensurável”. Segundo o mesmo autor, os principais recursos tangíveis são os recursos financeiros, os equipamentos, os imóveis e instalações, as terras, entre outros.

Do ponto de vista econômico, o enfoque deve ser dado aos resultados que o recurso pode trazer e não no ativo em si. Essa perspectiva é consistente com o método de fluxo de caixa descontando, onde o valor de um ativo (ou capital) é obtido pela soma dos fluxos de caixa futuros, descontados a uma taxa apropriada ao seu nível de risco. Perez e Famá (2006) mencionam que os ativos são recursos da empresa capazes de gerar benefícios futuros: entradas de caixa ou redução de saídas de caixa.

Os recursos tangíveis apresentam algumas limitações na sua utilização. Existe um limite para exploração de economias de escala e escopo. Para Besanko et al. (2006), as economias de escala são normalmente definidas em termos de funções de custo médio em declínio, enquanto as economias de escopo são definidas em termos de custo total relativo de produção, ao produzir dois ou mais produtos numa única unidade de produção, comparados à produção desses mesmos produtos em unidades separadas. Besanko et al. (2006) relatam que a fonte mais comum de economias de escala é a diluição de custos fixos.

Lev (2001), ao comentar as limitações dos ativos tangíveis, relata que podem ser alavancados para explorar economias de escala e escopo, mas até determinado limite. Essa limitação indica que os ativos tangíveis apresentam escalabilidade limitada, ou seja, beneficiam-se da ampliação do volume de produção até determinado limite; novas ampliações da produção requerem novos dispêndios de capital, que só se tornam viáveis quando o projeto proporciona valor presente líquido [VPL] positivo.

Outra limitação dos ativos tangíveis refere-se à rivalidade na sua utilização. Essa característica implica que o ativo não pode ser utilizado em duas alternativas ao mesmo tempo. Kayo (2002, p. 21) relata que "os ativos tangíveis são, por natureza, disputados por diferentes usos alternativos; é impossível utilizá-los simultaneamente em situações diferentes. Uma máquina não pode fabricar dois produtos diferentes simultaneamente”. Os ativos tangíveis têm potencial para criação de valor; no entanto, na perspectiva da RBV, a maioria desses ativos não contribui para a sustentabilidade do desempenho superior.

\section{Procedimentos METOdOLÓGICOS}

\section{Amostra e Dados}

A amostra foi constituída por empresas brasileiras de capital aberto com ações listadas na Bovespa (Bolsa de Valores de São Paulo) e com informações disponíveis na base de dados da Consultoria Economática ${ }^{\circledR}$. Utilizaram-se basicamente dados secundários, conforme classificação de Malhotra (2000), sendo extraídas as seguintes informações de cada empresa participante da amostra: ativo total [AT], patrimônio líquido [PL], dívidas totais, valor de mercado da firma e retorno sobre o ativo [ROA]. Os valores monetários foram deflacionados pelo Índice Geral de Preços-Disponibilidade Interna [IGP-DI] para 31 de dezembro de 2007.

Para a classificação setorial das firmas, adotou-se o critério proposto pela consultoria Economática ${ }^{\circledR}$. Seguindo os critérios de Villalonga (2004), não fizeram parte da amostra as empresas classificadas como bancos, fundos, clubes e confederações. Tais setores apresentam características operacionais e financeiras, que diferem das empresas industriais, que constituem a maior parte da amostra; por isso poderiam distorcer os resultados encontrados. Da mesma maneira, adotando critério 
semelhante ao utilizado por Fama e French (1993) e Daniel e Titman (2006), foram excluídas da amostra inicial empresas que apresentaram patrimônio líquido [PL] negativo em qualquer exercício correspondente ao período da pesquisa, ou seja, 1996 a 2007, por presumir-se que tenham sérios problemas de solvência financeira. Dessa forma, a amostra foi composta por empresas com dados válidos e disponíveis para o período compreendido entre 1996 e 2007, totalizando, assim, 228 empresas dos mais diversos setores da economia.

Para testar se a intangibilidade ou a tangibilidade dos recursos da firma influenciam no seu desempenho persistente, ou seja, se são fontes de vantagem ou desvantagem competitiva sustentável, foram extraídas duas subamostras da amostra completa. A primeira foi constituída por empresas que apresentaram retorno sobre o ativo [ROA] acima do ROA médio do setor em, pelo menos, quatro anos consecutivos. Essa subamostra foi composta por 125 empresas que apresentam vantagem competitiva sustentável. A segunda constituiu-se de empresas que apresentaram ROA abaixo do ROA médio do setor em pelo menos quatro anos consecutivos, totalizando 103 firmas.

\section{Modelos e Tratamento Estatístico dos Dados}

Para atender aos propósitos do estudo e responder ao problema de pesquisa levantado, utilizaram-se modelos adaptados aos propostos por Villalonga (2004) e Daniel e Titman (2006). Na análise da relação do desempenho persistente com a intangibilidade ou a tangibilidade dos recursos das firmas, foi utilizado o painel dinâmico com estimativas pelo Método de Momentos Generalizado [GMM] no modelo proposto por Arellano e Bond (1991).

O conceito de desempenho persistente se confunde com o conceito de vantagem competitiva sustentável e, de acordo com Bharadwaj et al. (1993) e Villalonga (2004), implica ter um desempenho superior por um longo prazo. Neste trabalho, o prazo considerado para garantir desempenho persistente correspondeu a quatro anos. De acordo com Rumelt (1991), esse prazo permite incluir os efeitos relativos a todo um ciclo de negócios. Na análise da relação do desempenho persistente com a intangibilidade, o modelo pode ser expresso por:

$$
\begin{aligned}
& \mathrm{LEF}_{\mathrm{it}}=\alpha_{\mathrm{i}}+\beta_{0} * \mathrm{LEF}_{\mathrm{it}-1}+\beta_{1} * \mathrm{Q}_{\mathrm{it}}+\sum_{\mathrm{j}=\mathrm{i}}^{\mathrm{j}} \beta_{2} * \mathrm{LEF}_{\mathrm{it}-1} * \mathrm{Q}_{\mathrm{it}} * \mathrm{Dj}+\mu_{\mathrm{i}}+\eta_{\mathrm{it}}, \operatorname{com} \mathrm{i}=1, \ldots, \mathrm{N} \\
& \text { e } \mathrm{t}=1, \ldots, \mathrm{T} \text {. }
\end{aligned}
$$

em que: $\mathrm{LEF}_{\mathrm{it}}=$ lucro específico da firma; $\mathrm{LEF}_{\mathrm{it}-1}=$ lucro específico da firma defasado; $\mathrm{Q}_{\mathrm{it}}=\mathrm{Q}$ de Tobin - variável de intangibilidade; $\mathrm{Dj}=$ são dummies para setores da economia $(j=17) ; \mu_{i}=$ efeito específico da firma que não varia com o tempo; $\eta_{\mathrm{it}}=$ termo de erro da firma; $\beta_{0}=$ captura a persistência do LEF; $\beta_{1}$ = captura o efeito da intangibilidade dos recursos no LEF; $\beta_{2 j}=$ captura o efeito da intangibilidade dos recursos na persistência do LEF nos setores da economia. Os subscritos i e t referem-se à empresa e ao tempo, respectivamente.

Na tentativa de explicar a influência da tangibilidade dos recursos da firma sobre o seu desempenho superior e persistente, utilizou-se uma variável adaptada do modelo proposto por Daniel e Titman (2006). Os autores afirmam que o retorno passado de uma firma pode ser decomposto em retorno devido às informações tangíveis e intangíveis. Definem como retorno tangível aquele que é resultante do crescimento das métricas, ou seja, quantificado pelo desempenho contábil. A variável de tangibilidade variação do book [VarBook], utilizada para mensurar a variação do patrimônio líquido, foi utilizada no novo modelo, expresso pela seguinte equação:

$$
\begin{aligned}
& \operatorname{LEF}_{\mathrm{it}}=\alpha_{\mathrm{i}}+\beta_{0} * \mathrm{LEF}_{\mathrm{it}-1}+\beta_{1} * \operatorname{VarBook}_{\mathrm{it}}+\sum_{\mathrm{i}=1}^{\mathrm{j}} \beta_{2} * \mathrm{LEF}_{\mathrm{it}-1} * \operatorname{VarBook}_{\mathrm{it}} * \mathrm{Dj}+\mu_{\mathrm{i}}+\eta_{\mathrm{it}}, \\
& \text { com } \mathrm{i}=1, \ldots, \mathrm{N} \text { e } \mathrm{t}=1, \ldots, \mathrm{T} \text {. }
\end{aligned}
$$


em que: $\mathrm{LEF}_{\text {it }}=$ lucro específico da firma; $\mathrm{LEF}_{\mathrm{it}-1}=$ lucro específico da firma defasado; $\operatorname{VarBook}_{\mathrm{it}}=$ variável de tangibilidade; calculada pelo logaritmo da variação do PL do ano t-1 para o ano t; $\mathrm{Dj}=$ são dummies para setores da economia $(j=17) ; \mu_{i}=$ efeito específico da firma que não varia com o tempo; $\eta_{\text {it }}=$ termo de erro da firma; $\beta_{0}=$ captura a persistência do LEF; $\beta_{1}=$ captura o efeito da tangibilidade dos recursos no LEF; $\beta_{2 \mathrm{j}}$ = captura o efeito da tangibilidade dos recursos na persistência do LEF nos setores da economia. Os subscritos $\mathrm{i}$ e $\mathrm{t}$ referem-se à empresa e ao tempo, respectivamente.

\section{Descrição e Operacionalização das Variáveis}

Lucro específico da firma [LEF]. O desempenho da firma é medido pelo LEF, conforme modelo da Villalonga (2004). É calculado pela diferença entre o retorno sobre o ativo [ROA] da firma, menos o valor médio do ROA do setor da economia do qual a empresa faz parte. Este procedimento foi realizado para cada ano considerado na pesquisa, de 1996 a 2007. Empresas que apresentaram valores missing para determinado ano foram excluídas do cálculo da média do ROA do setor para aquele ano, não implicando, contudo, a eliminação da empresa da amostra nos anos subsequentes. O LEF pode ser expresso pela equação:

$$
\mathrm{LEF}_{\mathrm{it}}=\mathrm{ROA}_{\mathrm{it}}-\left(\sum_{\mathrm{i}=1}^{\mathrm{Ijt}} \frac{\mathrm{ROA}_{\mathrm{ijt}}}{\mathrm{I}_{\mathrm{jt}}}\right) \text {, }
$$

em que: $\mathrm{I}_{\mathrm{jt}}$ corresponde ao número de firmas com dados disponíveis, do setor $\mathrm{j}$ no ano $\mathrm{t}$; $\mathrm{i}$ e $\mathrm{t}$ referemse à empresa e ao tempo, respectivamente.

Q de Tobin. Medida de intangibilidade da firma, conforme modelo proposto por Villalonga (2004). O Q de Tobin foi calculado pela aproximação proposta por Chung e Pruitt (1994), sendo definido por:

$$
\mathrm{QdeTobin}=\frac{\mathrm{VM}+\mathrm{DT}}{\mathrm{AT}}
$$

em que: VM - valor de mercado da firma; DT - dívidas totais contábeis da firma; AT - ativo total da empresa.

Variação da tangibilidade (VarBook). Medida de tangibilidade dos recursos da firma, adaptada do modelo proposto por Daniel e Titman (2006). O valor da variação do book (VarBook) da firma i no ano $t$ foi calculado considerando o logaritmo da variação do patrimônio líquido da firma i no ano t-1 para o ano t. Os valores da variável VarBook foram calculados para as empresas integrantes da amostra. Devido à indisponibilidade dos dados do ano de 1995, não foi possível calcular a variação tangível (VarBook) para o ano de 1996.

\section{ResUlTADOS DA PESQUISA}

\section{Influência da Intangibilidade no Desempenho Persistente das Firmas Brasileiras}

Utilizando-se as estimativas de Arellano-Bond de duas fases, percebe-se que o teste de Sargan (Tabela 1) não apresentou significância estatística em nenhuma das três amostras, não rejeitando assim a hipótese nula de que os instrumentos são válidos. Isso indica que o modelo utilizado está corretamente especificado e os instrumentos usados são válidos para as três amostras. 
Pela análise dos resultados obtidos da amostra completa (Tabela 1), percebe-se que o coeficiente que mede a persistência do desempenho superior (DLEF-1) não apresentou significância estatística. Isso indica que a porcentagem do total do lucro específico da firma em qualquer período antes do período $\mathrm{t}$ que permanece no período t não é significativa estatisticamente.

Quando se analisam os setores separadamente, percebe-se que todos os coeficientes $\beta_{2 j}$ das interações (Q x LEFDef x DSetorial) que capturam o efeito da intangibilidade dos recursos na persistência do LEF nos setores da economia apresentaram sinal negativo com significância estatística, com exceção do setor de mineração, que também apresentou sinal negativo, mas não foi significativo estatisticamente. Esses resultados mostram que quanto maior o Q de Tobin, ou seja, a intangibilidade dos ativos das firmas desses setores, menor a persistência de seus lucros específicos, indicando que a intangibilidade dos recursos não é um fator que contribui para a manutenção do desempenho superior das empresas brasileiras, ou seja, não promove vantagem competitiva sustentável das firmas brasileiras de capital aberto.

Considerando ainda a análise dos setores, percebe-se que o setor que teve o desempenho persistente mais influenciado negativamente pela intangibilidade dos recursos foi o de construção, enquanto o menos afetado foi o de comércio. Observando-se a variação nos valores dos coeficientes $\beta_{2 \mathrm{j}}$, bem como seus níveis de significância, fica evidente que a intangibilidade influencia, de forma diferenciada, os diversos setores da economia. No entanto, devido ao sinal negativo apresentado para todos os $\beta_{2 \mathrm{j}}$, sugere-se que a intangibilidade dos recursos contribui para reduzir, de forma diferenciada, nos setores da economia, a persistência do desempenho superior das firmas.

Os resultados obtidos para as subamostras são semelhantes aos obtidos para a amostra completa, em que todos os coeficientes $\beta_{2 \mathrm{j}}$ também apresentaram sinal negativo; no entanto existem diferenças na forma de interpretação desses resultados. Para a subamostra vantagem competitiva, que contempla empresas com desempenho acima da média em pelo menos quatro anos consecutivos, o sinal negativo dos coeficientes $\beta_{2 j}$ indica que a intangibilidade dos recursos contribui para a redução da persistência do desempenho superior. Para a subamostra desvantagem competitiva, que contempla empresas com desempenho abaixo da média em pelo menos quatro anos consecutivos, o sinal negativo dos coeficientes $\beta_{2 \mathrm{j}}$ indica que a intangibilidade dos recursos contribui para a redução da persistência das perdas específicas da firma. 
Tabela 1

Influência do Q de Tobin na persistência do Lucro Específico da Firma [LEF] - Período 1996 a 2007

\begin{tabular}{|c|c|c|c|c|c|c|}
\hline & \multicolumn{2}{|l|}{ Amostra completa } & \multicolumn{2}{|c|}{ Vantagem competitiva } & \multicolumn{2}{|c|}{ Desvantagem competitiva } \\
\hline & Coeficiente & Erro Padrão & Coeficiente & Erro Padrão & Coeficiente & Erro Padrão \\
\hline Intercepto & $-1,36209 * * *$ & 0,3197 & $-0,79183$ & 0,3826 & $-1,52492 * * *$ & 0,3720 \\
\hline $\operatorname{DLEF}(-1)$ & 0,01326 & 0,0442 & $0,16999 * *$ & 0,0684 & 0,09809 & 0,0630 \\
\hline Q de Tobin & $2,07901^{* * *}$ & 0,4898 & $1,86968 * * *$ & 0,5165 & 0,89394 & 0,5932 \\
\hline Q X LEFDef X D(AgroPesc) & $-0,52743 * * *$ & 0,1125 & $-0,24146$ & 0,7244 & $-0,60530 * * *$ & 0,2190 \\
\hline Q X LEFDef X D(Alim) & $-0,29753 * * *$ & 0,1015 & $-0,25897 * * *$ & 0,0635 & $-0,46947 * * *$ & 0,0839 \\
\hline Q X LEFDef X D(Com) & $-0,08421 * * *$ & 0,0186 & $-0,08656 * * *$ & 0,0194 & $-0,30619 *$ & 0,1725 \\
\hline Q X LEFDef X D(Constr) & $-1,98600 * * *$ & 0,4266 & $-2,41661^{* *}$ & 1,0612 & $-1,34732 *$ & 0,6944 \\
\hline Q X LEFDef X D(Eletr) & $-0,35158 * * *$ & 0,0655 & $-0,35047 * * *$ & 0,0857 & $-0,44783 * * *$ & 0,1384 \\
\hline Q X LEFDef X D(EnElet) & $-0,23374 *$ & 0,1359 & $-0,23672 *$ & 0,1267 & $-0,56621 * *$ & 0,2565 \\
\hline Q X LEFDef X D(MaqInd) & $-0,43646 * * *$ & 0,0474 & $-0,50657 * * *$ & 0,0416 & $-0,29540 * *$ & 0,1212 \\
\hline Q X LEFDef X D(Miner) & $-0,08585$ & 0,1279 & $-0,45385 * * *$ & 0,1172 & $-0,04529$ & 0,0512 \\
\hline Q X LEFDef X D(PapCel) & $-0,53493 * * *$ & 0,1523 & $-0,41411 * * *$ & 0,0698 & $-0,94583 * * *$ & 0,1085 \\
\hline Q X LEFDef X D(PetrGas) & $-0,49993 * * *$ & 0,1790 & $-0,43084 * * *$ & 0,0847 & $-0,80817^{*}$ & 0,4555 \\
\hline Q X LEFDef X D(Quim) & $-0,49562 * * *$ & 0,1621 & $-0,24542 *$ & 0,1320 & $-0,93269 * * *$ & 0,1879 \\
\hline Q X LEFDef X D(SidMet) & $-0,24342 *$ & 0,1421 & $-0,21526 * *$ & 0,0874 & $-0,70957 * * *$ & 0,1602 \\
\hline Q X LEFDef X D(Telec) & $-0,23625 * * *$ & 0,0605 & $-0,26509 * * *$ & 0,0616 & $-0,17503$ & 0,1169 \\
\hline Q X LEFDef X D(Têxtil) & $-0,36615 * * *$ & 0,1218 & $-0,40139 * * *$ & 0,1182 & $-0,53020$ & 0,3443 \\
\hline Q X LEFDef X D(TranspSer) & $-0,19554 * * *$ & 0,0594 & $-0,18247$ & 0,1137 & - & - \\
\hline Q X LEFDef X D(VeicPec) & $-0,41776 * * *$ & 0,0705 & $-0,42886 * * *$ & 0,0774 & $-0,29347 * * *$ & 0,0942 \\
\hline Teste de Sargan (prob) & 0,2899 & & 0,2909 & & 0,2289 & \\
\hline Teste (prob) - $\mathrm{AR}^{2}$ & 0,2956 & & 0,5277 & & 0,9109 & \\
\hline Número de Oservações & 1756 & & 1012 & & 828 & \\
\hline
\end{tabular}

Nota. Variável dependente: Lucro Específico da Firma [LEF]. Estimação por Método dos Momentos Generalizado [GMM].

Fonte: elaborado pelo autor.

* Significativo a $10 \%$.; ** Significativo a 5\%.; *** Significativo a $1 \%$. 
Os resultados obtidos para as firmas que apresentavam vantagem competitiva sugerem que a intangibilidade dos recursos contribuiu significativamente para reduzir a persistência do desempenho superior em todos os setores estudados, com exceção dos setores agro/pesca e transporte/serviços, que também apresentaram coeficientes $\beta_{2 j}$ negativos, porém sem significância estatística. Por outro lado, para as firmas que apresentavam desvantagem competitiva, a intangibilidade dos recursos contribui de forma significativa para reduzir a persistência das perdas em todos os setores, com exceção dos setores de mineração, telecomunicações e têxtil, que também apresentaram coeficientes $\beta_{2 j}$ negativos, porém sem significância estatística.

Diferenças podem ser observadas nos resultados das duas subamostras. Na subamostra vantagem competitiva, percebe-se que o coeficiente $\beta_{0}$, que mede a persistência do desempenho superior (DLEF-1), apresentou significância estatística de 5\%. Na subamostra desvantagem competitiva não foi percebida significância estatística para esse coeficiente. Outra diferença observada diz respeito ao nível de significância da variável que mede a intangibilidade, o Q de Tobin. Na subamostra vantagem competitiva, ela se mostrou altamente significativa, enquanto na subamostra desvantagem competitiva não foi observada significância estatística. Estes resultados indicam que a relação entre a intangibilidade e a persistência do desempenho superior é mais robusta na subamostra que contempla firmas com desempenho acima da média. Essa robustez pode ser percebida também entre os setores da economia em que, na subamostra que contempla firmas com desvantagem competitiva, vários setores não apresentaram significância estatística para a relação entre intangibilidade e persistência do desempenho.

Os resultados apresentados no presente artigo diferem dos obtidos por Villalonga (2004), que analisou empresas norte-americanas. Villalonga (2004) verificou que, de maneira geral, a intangibilidade dos recursos desempenha papel importante para a persistência do desempenho superior das firmas, confirmando, assim, os pressupostos da RBV. Sua conclusão é construída com base na constatação de que a variável de interação do Q de Tobin com o lucro específico defasado (variável independente) apresenta relação positiva e significante com o lucro específico contemporâneo (variável dependente). Assim, quanto maior a intangibilidade e lucro específico de uma firma no ano anterior, maior seu lucro específico no ano corrente. Além disso, esse resultado se repete para quase todos os setores incluídos em sua pesquisa, indicando que a intangibilidade é fonte de vantagem competitiva sustentável das firmas dos Estados Unidos.

\section{Influência da Tangibilidade no Desempenho Persistente das Firmas Brasileiras}

Utilizando-se as estimativas de Arellano-Bond de duas fases, percebe-se que o teste de Sargan (Tabela 2) apresentou significância estatística de $10 \%$ para as amostras completa e vantagem competitiva, rejeitando, assim, a hipótese nula de que os instrumentos são válidos. Alves e Bruno (2006) se depararam com a mesma situação na estimação via GMM nos modelos Arellano-Bond e relatam que a rejeição da hipótese nula do teste de Sargan não necessariamente implica a rejeição do modelo.

O teste de autocorrelação $\left(\mathrm{AR}^{2}\right)$ aceitou a hipótese nula de ausência de autocorrelação de segunda ordem, tendo obtido p-valor de 0,4066 para a amostra completa; 0,9100 para a amostra vantagem competitiva; e 0,6779 para a amostra desvantagem competitiva, mostrando, assim, indícios de validade do modelo. 
Tabela 2

Influência da Variação Tangível na Persistência do Lucro Específico da Firma [LEF] - Período 1996 a 2007

\begin{tabular}{|c|c|c|c|c|c|c|}
\hline & \multicolumn{2}{|c|}{ Amostra completa } & \multicolumn{2}{|c|}{ Vantagem competitiva } & \multicolumn{2}{|c|}{ Desvantagem competitiva } \\
\hline & Coeficiente & Erro Padrão & Coeficiente & Erro Padrão & Coeficiente & Erro Padrão \\
\hline Intercepto & 0,00559 & 0,1094 & $-0,01775$ & 0,1244 & 0,04329 & 0,1507 \\
\hline $\operatorname{DLEF}(-1)$ & $0,23707^{* * *}$ & 0,0572 & $0,21639 * * *$ & 0,0733 & $0,24993 * * *$ & 0,0604 \\
\hline VarBook & $5,04499 * * *$ & 0,8506 & $6,74083^{* * *}$ & 1,1376 & $5,03653 * * *$ & 1,3454 \\
\hline VarBook X LEFDef X D(AgrPes) & $0,86070^{* * *}$ & 0,2482 & $8,13412^{* *}$ & 3,3455 & $0,81946^{* * *}$ & 0,1953 \\
\hline VarBook X LEFDef X D(Alim) & $-0,83597 * *$ & 0,3370 & $-1,67156^{* * *}$ & 0,4434 & $-0,21765$ & 0,6520 \\
\hline VarBook X LEFDef X D(Com) & $-0,13143$ & 0,3174 & $-0,48497 * * *$ & 0,1343 & $-0,16316$ & 0,3934 \\
\hline VarBook X LEFDef X D(Constr) & 0,99700 & 2,1391 & $-1,15834$ & 6,8592 & $-0,33934$ & 0,6455 \\
\hline VarBook X LEFDef X D(Eletr) & $3,42016^{* *}$ & 1,5704 & 0,98428 & 0,7926 & $1,53671^{* *}$ & 0,7506 \\
\hline VarBook X LEFDef X D(EnElet) & $-0,94700 * *$ & 0,4702 & $-1,22603^{* *}$ & 0,5980 & $-0,95998$ & 1,0923 \\
\hline VarBook X LEFDef X D(MqInd) & $2,39568 *$ & 1,3336 & 0,64719 & 1,3145 & $3,15192 * * *$ & 1,0932 \\
\hline VarBook X LEFDef X D(Miner) & $-0,07963$ & 0,1717 & $-1,65862 * * *$ & 0,6255 & $-0,02921$ & 0,1771 \\
\hline VarBook X LEFDef X D(PapCel) & $-0,30262$ & 1,5515 & $0,53694^{*}$ & 0,3138 & $-3,05513$ & 1,9800 \\
\hline VarBook X LEFDef X D(PetrGa) & $-1,01374 * * *$ & 0,3906 & $-1,38921^{* * *}$ & 0,2643 & $-0,99921 * * *$ & 0,3340 \\
\hline VarBook X LEFDef X D(Quim) & 0,08988 & 0,4339 & $-0,18258$ & 1,2760 & 0,19683 & 0,2559 \\
\hline VarBook X LEFDef X D(SidMet) & $-0,45879$ & 0,3105 & $-1,09541 * * *$ & 0,1757 & $-0,11522$ & 0,2730 \\
\hline VarBook X LEFDef X D(Telec) & $-0,78478 * * *$ & 0,2222 & $-1,41541^{* * *}$ & 0,4139 & $-0,61839 * *$ & 0,2594 \\
\hline VarBook X LEFDef X D(Têxtil) & 0,03310 & 0,2653 & 1,64881 & 2,6235 & 0,04294 & 0,2776 \\
\hline VarBook X LEFDef X D(TranSe) & $-1,12407 * * *$ & 0,3103 & $-1,34646^{* * * *}$ & 0,4206 & - & - \\
\hline VarBookX LEFDef X D(VeicPe) & $0,51576 * * *$ & 0,11175 & $-0,75248$ & 0,5810 & $1,83834 * * *$ & 0,3453 \\
\hline Teste de Sargan (prob) & 0,0901 & & 0,0640 & & 0,1478 & \\
\hline Teste (prob) - $\mathrm{AR}^{2}$ & 0,4066 & & 0,9100 & & 0,6779 & \\
\hline Número de Oservações & 1744 & & 1006 & & 818 & \\
\hline
\end{tabular}

Nota. Variável dependente: Lucro Específico da Firma [LEF]. Método de estimação por Método dos Momentos Generalizado [GMM].

Fonte: elaborado pelo autor.

* Significativo a $10 \%$.; ** Significativo a 5\%.; *** Significativo a $1 \%$. 
Analisando-se, na Tabela 2, os resultados obtidos, percebe-se que o coeficiente que mede a persistência do desempenho superior (DLEF-1) apresentou alta significância estatística para a amostra completa. Isso indica que a porcentagem do total do lucro específico da firma em qualquer período antes do período t que permanece no período t é significativa estatisticamente. A persistência média observada foi de 23,70\%, conforme coeficiente da variável DLEF(-1) na amostra completa. Pode-se observar ainda, por meio do coeficiente da variável VarBook, que o efeito da tangibilidade dos recursos no nível do lucro específico da firma é positivo e altamente significativo nas três amostras.

A influência da tangibilidade dos recursos na persistência do lucro específico da firma nos diferentes setores da economia, pode ser observada por meio dos coeficientes $\beta_{2 j}$ das interações ( $Q$ x LEFDef x DSetorial). Na amostra completa percebe-se que os setores agro/ pesca, eletrônica, máquinas industriais e veículos/peças apresentaram sinais positivos e significância estatística para os coeficientes $\beta_{2 \mathrm{j}}$. Esses resultados sugerem que quanto maior a tangibilidade dos ativos das firmas desses setores, maior a persistência de seus lucros específicos, indicando que a tangibilidade dos recursos é fator que contribui para a manutenção do desempenho superior das empresas desses setores. Por outro lado, percebe-se que nos setores alimentação, energia elétrica, petróleo/gás, telecomunicações e transporte/serviços os sinais dos coeficientes $\beta_{2 j}$ foram negativos com significância estatística, indicando que quanto maior a tangibilidade dos recursos das firmas desses setores, menor a persistência do lucro específico. Embora em menor número, firmas de vários setores apresentaram influência positiva da tangibilidade dos ativos no seu desempenho persistente, sugerindo, assim, que a tangibilidade, medida pela variação do PL, promove vantagem competitiva sustentável das firmas desses setores.

Pelos dados da Tabela 2, observa-se, por meio dos coeficientes $\beta_{2 \mathrm{j}}$, que a influência da tangibilidade no desempenho persistente das firmas brasileiras difere entre os setores da economia. A variação no sinal, nos valores dos coeficientes e nos níveis de significância, evidencia a influência da tangibilidade de forma diferenciada nos diversos setores. Percebe-se que, em alguns setores, o sinal do coeficiente $\beta_{2 \mathrm{j}}$ é positivo e significativo, sugerindo que a tangibilidade dos recursos contribui, de forma positiva e significativa, para a persistência do desempenho acima da média, enquanto em outros setores a influência é negativa e significativa, sugerindo que a tangibilidade contribui para reduzir a persistência do desempenho superior das firmas.

Os resultados obtidos para a subamostra vantagem competitiva sugerem que a tangibilidade dos recursos, medida pela variação do PL, tem relação negativa com o desempenho persistente para a maioria dos setores estudados. Isto pode ser percebido por meio do sinal negativo com significância estatística dos coeficientes $\beta_{2 \mathrm{j}}$ dos setores de alimentação, comércio, energia elétrica, mineração, petróleo/gás, siderurgia/metalurgia, telecomunicações e transporte/serviços, indicando que a tangibilidade dos recursos contribui para a redução da persistência do desempenho superior das firmas desses setores. Percebe-se que apenas os setores agro/pesca e papel/celulose apresentaram relação positiva e significativa, indicando que, quanto maior a tangibilidade dos recursos desses setores, maior será a persistência do desempenho superior das firmas. Para a subamostra desvantagem competitiva, os setores agro/pesca, eletrônica, máquinas industriais e veículos/peças apresentaram sinal positivo com significância estatística para os coeficientes $\beta_{2 \mathrm{j}}$, indicando que a tangibilidade dos recursos contribui para a persistência das perdas específicas das firmas desses setores. Nos setores de petróleo/gás e telecomunicações, a tangibilidade dos recursos contribui para reduzir a persistência das perdas específicas das firmas desses setores.

Diante dos resultados expostos, percebe-se que a relação da tangibilidade dos recursos com o desempenho persistente das firmas é bastante variável entre os setores da economia. Entretanto existem evidências estatísticas de que a variação tangível, devida à variação patrimonial, é fator que contribui, de maneira relevante, para a sustentabilidade do desempenho superior das firmas dos setores agro/pesca, eletrônica, máquinas industriais e veículos/peças. Dessa forma, existem indícios de que 
investimentos em capital tangível não só contribuem para o desempenho superior, mas também são relevantes para a sustentabilidade do desempenho superior para as firmas desses setores.

\section{CONSIDERAÇÕES FINAIS}

Os resultados encontrados na pesquisa empírica trazem algumas implicações teóricas e gerenciais que merecem ser relatadas. No aspecto teórico, preconiza a RBV que os recursos sejam distribuídos de forma heterogênea entre as firmas; aqueles que são específicos, raros e de difícil imitação têm o potencial para promover o desempenho superior e sustentável. Nesse sentido, esperava-se que os recursos intangíveis, devido às suas características e especificidades, apresentassem relação positiva e significativa com o desempenho superior e persistente, promovendo, assim, vantagem competitiva sustentável. Não foi esse o resultado encontrado na realidade brasileira. Em vários setores da economia predominaram os recursos tangíveis como fonte de vantagem competitiva sustentável.

Uma dúvida que pode derivar desses resultados: é o porquê esse fenômeno é observado no Brasil, isto é, por que os resultados do presente artigo mostraram que no Brasil a intangibilidade não afeta positivamente o desempenho persistente das firmas? Uma possível explicação para essa diferença em relação às empresas internacionais, particularmente as norte-americanas, seja a natureza das empresas desses diferentes países. Nos Estados Unidos, muitas empresas destinam vultosos recursos financeiros à pesquisa e ao desenvolvimento de novos produtos, gerando valiosos recursos de inovação que, por sua vez, geram fluxos de caixa significativos no futuro. Isso tem levado o valor de mercado das empresas norte-americanas a uma crescente valorização. No Brasil, por outro lado, grande parte das empresas de capital aberto (e, portanto, passíveis de análise em função da disponibilidade de dados) atua em setores mais intensivos em capital tangível do que intangível. Como resultado, a diferença entre o valor de mercado e o valor contábil das firmas é, em média, menor do que o encontrado em empresas dos Estados Unidos.

No aspecto gerencial, os resultados obtidos podem servir para nortear os gestores nas suas decisões de investimento. Em determinados setores da economia, os investimentos em ativos físicos, tais como máquinas, equipamentos, construções, entre outros, parecem ser mais adequados para a promoção do desempenho superior e persistente das firmas do que investimentos em recursos intangíveis, como marcas, patentes, propagandas, entre outros.

Entretanto novas investigações precisam ser realizadas para a confirmação desses resultados, principalmente devido à baixa liquidez de muitas ações de firmas integrantes da amostra. Esta, a propósito, é uma das limitações do presente artigo. Como algumas variáveis do modelo testado utilizaram o valor de mercado da firma, a baixa liquidez pode provocar distorções no preço das ações das empresas analisadas e, consequentemente, prejudicar a análise e a interpretação dos resultados. Uma segunda limitação diz respeito à variável utilizada para representar a intangibilidade $(\mathrm{Q}$ de Tobin), que mede a relação entre valor de mercado e ativo contábil. Esta variável pode, teoricamente, representar outros fatores que não apenas o efeito dos ativos intangíveis. Por exemplo, o Q de Tobin pode indicar, como lembram Famá e Barros (2000), oportunidade de novos investimentos ou poder de monopólio. Além disso, o valor de mercado (numerador do Q de Tobin) é muito sensível a fatores macroeconômicas ou outras oscilações conjunturais que podem afetar o preço das ações no curto prazo, mas não necessariamente implicam a redução do valor intrínseco da firma no longo prazo. Uma terceira limitação diz respeito à formação da amostra. A maior parte das empresas que fizeram parte desta pesquisa é formada por empresas de capital aberto que atuam em setores tradicionais da economia (como, por exemplo, siderurgia, metalurgia, petróleo etc.) e, portanto, intensivas em capital. Muitas empresas que supostamente representariam setores mais inovadores (como o de tecnologia de informação) não entraram na amostra ou porque abriram o capital recentemente (e seus dados eram insuficientes) ou porque, simplesmente, não eram de capital aberto e, portanto, não eram obrigadas a divulgar suas demonstrações financeiras. 
Para pesquisas futuras, sugere-se a adoção de medidas alternativas de desempenho, bem como de mensuração dos recursos tangíveis e intangíveis. Adicionalmente, a expansão da amostra, com a inclusão de empresas representativas de setores mais inovadores, pode apresentar resultados mais próximos aos encontrados nas pesquisas de países mais desenvolvidos.

\section{Artigo recebido em 10.07.2009. Aprovado em 03.02.2010.}

\section{REFERÊNCIAS BIBLIOGRÁFICAS}

Acquaah, M. (2003). Corporate management, industry competition and the sustainability of firm abnormal profitability. Journal of Management and Governance, 7(1), 57-85.

Ali Shah, S. Z., \& Akbar, S. (2008). Value relevance of advertising expenditure: a review of the literature. International Journal of Management Reviews, 10(4), 301-325.

Alves, P. F., \& Bruno, M. A. P. (2006). Crescimento das indústrias exportadoras no Brasil: um modelo dinâmico de efeitos fixos com o PIB mundial como variável. [Texto para Discussão, $\mathrm{N}^{\circ}$ 1231]. Brasília: Ministério do Planejamento, Orçamento e Gestão/Instituto de Pesquisa Econômica Aplicada.

Amit, R., \& Schoemaker, P. J. (1993). Strategic assets and organizational rent. Strategic Management Journal, 14(1), 33-46.

Antunes, M. A., \& Procianoy, J. L. (2003). Os efeitos das decisões de investimento das empresas sobre os preços de suas ações no mercado de capitais. Revista de Administração da USP, 38(1), $5-14$.

Arellano, M., \& Bond, S. (1991). Some tests of specification for panel data: Monte Carlo evidence and an application to employment equations. Review of Economic Studies, 58(2), 277-297.

Barney, J. (1991). Firm resources and sustained competitive advantage. Journal of Management, 17(1), 99-120.

Barney, J. (2002). Gaining and sustaining competitive advantage. Upper Saddle River: Prentice Hall.

Barth, M. E., Clement, M. B., Foster, G., \& Kasznik, R. (1998). Brand values and capital market valuation. Review of Accounting Studies, 3(1/2), 41-68.

Besanko, D., Dranove, D., Shanley, M., \& Schaefer, S. (2006). A economia da estratégia. Porto Alegre: Bookman.

Bharadwaj, S. G., Varadarajan, P. R, \& Fahy, J. (1993). Sustainable competitive advantage in service industries: a conceptual model and research propositions. Journal of Marketing, 57(1), 83-99.

Brito, L. A. L., \& Vasconcelos, F. C. de (2004a). A heterogeneidade do desempenho, suas causas e o conceito de vantagem competitiva: proposta de uma métrica. Revista de Administração Contemporânea, 8(Edição Especial), 107-129.

Brito, L. A. L., \& Vasconcelos, F. C. de (2004b). Vantagem competitiva: o construto e a métrica. Revista de Administração de Empresas, 44(2), 70-82.

Carneiro, J. M. T., \& Dib, L. A. (2006). Building a better measure of business performance. Anais do Encontro Nacional da Associação Nacional de Pós-Graduação e Pesquisa em Administração, Salvador, BA, Brasil, 30. 
Caves, R. E. (1984). Economic analyses end the quest for competitive advantage. The American Economic Review, 74(2), 127-132.

Chauvin, K. W., \& Hirschey, M. (1993). Advertising, R\&D expenditures and the market value of the firm. Financial Management, 22(4), 128-140.

Chung, K. H., \& Pruitt, S. W. (1994). A simple approximation of Tobin’s Q. Financial Management, 23(3), 70-74.

Connolly, R. A., \& Hirschey, M. (2005). Firm size and the effect of R\&D on Tobin's q. $R \& D$ Management, 35(2), 217-223.

Daniel, K., \& Titman, S. (2006). Market reactions to tangible and intangible information. The Journal of Finance, 59(4), 1605-1643.

Danthine, J. P., \& Jin, X. (2007). Intangible capital, corporate valuation and asset pricing. Economic Theory, 32(1), 157-177.

El-Shishini, H. (2001). Integrating financial and non-financial performance measures: state of art and research opportunities. Management Accouting Research Group Conference, Birmingham, UK.

Famá, R., \& Barros, L. A. B. C. (2000). Q de Tobin e seu uso em finanças: aspectos metodológicos e conceituais. Cadernos de Pesquisas em Administração, 7(4), 27-43.

Fama, E. F., \& French, K. R. (1993). Common risk factors in the returns on stocks and bonds. Journal of Financial Economics, 33(1/4), 3-56.

Ghemawat, P., \& Rivkin, J. (1999). Strategy and the business landscape. Upper Saddle River: Addison-Wesley, Reading.

Glick, W. H, Washburn, N. T., \& Miller, C. C. (2005). (2005, August). The myth of firm performance. Proceedings of the Annual Meeting of the Academy of Management, Honolulu, HI, USA, 70.

Helfat, E., \& Peteraf, M. A. (2003). Why is there a resourced-based view? Toward a theory of competitive heterogeneity. Strategic Management Journal, 24(10), 997-1010.

Kayo, E. K. (2002). A estrutura de capital e o risco das empresas tangível e intangível-intensivas: uma contribuição ao estudo da valoração de empresas. Tese de doutorado, Universidade de São Paulo, São Paulo, SP, Brasil.

Keller, K. L. (1998). Strategic brand management: building, measuring, and managing brand equity. New Jersey: Prentice Hall.

Lamb, R. (1993). A decisão de investimento nas empresas e a reação de mercado de capitais: um abordagem informacional. Dissertação de mestrado, Universidade Federal do Rio Grande do Sul, Porto Alegre, RS, Brasil.

Lee, R. P., \& Chen, Q. M. (2009). The immediate impact of new product introductions on stock price: the role of firm resources and size. Journal of Product Innovation Management, 26(1), 97-107.

Lev, B. (2001). Intangibles: management, measurement and reporting. Brookings Institution.

Lucchesi, E. P., \& Famá, R. (2007). O impacto das decisões de investimento das empresas no valor de mercado das ações negociadas na Bovespa no período de 1996 a 2003. Revista de Administração USP, 42(2), 249-260.

Malhotra, N. (2000). Pesquisa de marketing: uma orientação aplicada. Porto Alegre: Bookman. 
March, J. G., \& Sutton, R. I. (1997). Organizational performance as a dependent variable. Organization Science, 8(6), 698-706.

Martins, E. (1972). Contribuição à avaliação do ativo intangível. Tese de doutorado, Universidade de São Paulo, São Paulo, SP, Brasil.

Mathews, J. A. (2002). A resource-based view of Schumpeterian economic dynamics. Journal of Evolutionary Economics, 12(1/2), 29-54.

McConnell, J. J., \& Muscarella, C. J. (1985). Corporate capital expenditure decisions and the market value of the firm. Journal of Financial Economics, 14(3), 399-422.

Megna, P., \& Klock, M. (1993). The impact of intangible capital on Tobin's q in the semiconductor industry. The American Economic Review, 83(2), 265-269.

Morbey, G. K., \& Reithner, R. M. (1990). How R\&D affects sales growth, productivity and profitability. Research Technology Management, 33(3), 11-14.

Omaki, E. T. (2005). Recursos intangíveis e desempenho em grandes empresas brasileiras: avaliações dos recursos intangíveis como estimador de medidas de desempenho financeiras. Anais do Encontro Nacional da Associação Nacional de Pós-Graduação e Pesquisa em Administração, Brasília, DF, Brasil, 27.

Penrose, E. T. (1959). The theory of the growth of the firm. Oxford: Oxford University.

Perez, M. M., \& Famá, R. (2006). Ativos intangíveis e o desempenho empresarial. Revista Contabilidade e Finanças (40), 7-24.

Peteraf, M. A. (1993). The cornerstones of competitive advantage: a resource-based view. Strategic Management Journal, 14(3), 179-191.

Pindyck, R. S., \& Rubinfeld, D. L. (1994). Microeconomia. São Paulo: Makron Books.

Rugman, A. M., \& Verbeke, A. (2002). Edith Penrose's contribution to the resource-based view of strategic management. Strategic Management Journal, 23(8), 769-780.

Rumelt, R. P. (1991). How much does industry matter? Strategic Management Journal, 12(3), 167-185.

Rumelt, R. P. (2003). What in the world is competitive advantage? [Policy Working Paper $\mathrm{N}^{\circ}$ 2003105]. University of California, Los Angeles, Los Angeles, CA, USA.

Saraiva, R. S. (2007). Recursos e capabilidades estratégicos na indústria moveleira de Bento Gonçalves - RS. Dissertação de mestrado, Universidade Federal do Rio Grande do Sul, Porto Alegre, RS, Brasil.

Schmalensee, R. (1985). Do markets differ much? The American Economic Review, 75(3), 341-351.

Villalonga, B. (2004). Intangible resources, Tobin's q, and sustainability of performance differences. Journal of Economic Behavior \& Organization, 54(2), 205-230.

Yong, S. D., \& O’Byrne, S. F. (2003). Eva e gestão baseada em valor: guia prático para implementação. Porto Alegre: Bookman.

Wernerfelt, B. (1984). A resource-based view of the firm. Strategic Management Journal, 5(2), 171-180.

Wilk, E. O. de (2006). A relação entre estratégias, recursos e performance: uma investigação em empresas de vinhos finos do Cluster da Serra Gaúcha. Tese de doutorado, Universidade Federal do Rio Grande do Sul, Porto Alegre, RS, Brasil. 